\title{
Serum Fatty Acid Binding Protein 4 in Patients with Androgenetic Alopecia
}

A.I.El-Taweel ${ }^{1}$, A. I.Mustafa ${ }^{1}$, W.A.Abd El-Halim ${ }^{2}$ and S.A.Abdel-Fattah ${ }^{3}$

${ }^{1}$ Dermatology, Venereology and Andrology Dept., Faculty of Medicine, Benha Univ., Benha, Egypt

${ }^{2}$ Clinical and Chemical Pathology Dept., Faculty of Medicine, Benha Univ., Benha, Egypt

${ }^{3}$ Dermatology, Venereology and Andrology Dept., Faculty of Medicine, Zagazig Univ., Zagazig, Egypt

E-Mail:Sara@gmail.com

\begin{abstract}
Androgenetic alopecia (AGA) is the most common cause of hair loss. Adipocyte fatty acid-binding protein (FABP4), also termed adipocyte protein 2 (aP2), is an adipokine synthesized and released predominantly from adipocytes and, to a lesser extent, produced in macrophages and endothelial cells. Elevated levels of FABP4 are closely linked with the development of obesity, DM, hypertension and atherosclerosis. we aimed to assess the level of serum FABP4 in patients with AGA. This case-control study was conducted on thirty male patients and twenty female patients complaining of androgenetic alopecia, and thirty age and BMI matched healthy volunteers. Patients were recruited from outpatient clinic of Dermatology, Venereology and Andrology Department of Benha University Hospitals during the period from December 2017 to June 2018. All participants were tested for determination of fatty acid binding protein 4 serum levels. AGA patients showed significantly higher FABP4 levels when compared to controls. Serum FABP4 level was significantly higher in AGA patients than in control group so it might play a role in androgenetic alopecia pathogenesis. Moreover, it could be considered a biomarker for early diagnosis of the disease with high sensitivity, specificity and accuracy rate.
\end{abstract}

Keywords: Androgenetic alopecia, FABP4, Diagnosis .

\section{Introduction}

Androgenetic alopecia (AGA) is the most common cause of hair loss. Although it is a medically benign condition, it can have a significant psychosocial impact on patients. It is a very common condition encountered in almost all dermatology outpatient departments across the world [1].

Male pattern baldness is found to be associated with coronary artery disease, hypertension, insulin resistance, abnormal serum lipid profile, obesity, prostate cancer, benign prostatic hyperplasia, scalp pain, and smoking; high androgen levels can contribute to the development of atherosclerosis and thrombosis leading to hypercholesterolemia and hypertension [2].

Adipocyte fatty acid-binding protein (FABP4), also termed adipocyte protein 2 (aP2), is an adipokine synthesized and released predominantly from adipocytes and, to a lesser extent, produced in macrophages and endothelial cells. Elevated levels of FABP4 are closely linked with the development of obesity, DM, hypertension and atherosclerosis [3].

In the present study, we aimed to assess the level of serum FABP4 in patients with AGA.

\section{Patients and methods \\ 2.1 Patients}

This case-control study was conducted on thirty male patients and twenty female patients complaining of androgenetic alopecia, and thirty age and BMI matched healthy volunteers. Patients were recruited from outpatient clinic of Dermatology, Venereology and Andrology Department of Benha University Hospitals during the period from December 2017 to June 2018.

\subsubsection{Inclusion criteria}

The inclusion criteria for patients with AGA were: Different degrees of severity of androgenetic alopecia according to Hamilton Norwood classification (1975) and Ludwig scale, being over 18 years of age, and being willing to participate in the study.

\subsubsection{Administrative design}

This study was approved by the Research Ethical Committee of Benha Faculty of Medicine.

\subsubsection{Ethical consideration}

Before taking blood samples, an informed consent was taken from each subject included in the study.

\section{All participants were divided into two groups}

- Group A: Fifty patients with AGA.

- Group B: Thirty age, sex and BMI matched volunteers as controls group.

\subsection{Methods}

All patients were subjected to the following:

- Full history taking

- Clinical Examination

- Measure of the body height and weight to calculate the body mass index

\subsubsection{Laboratory investigations}

- All participants were tested for determination of fatty acid binding protein 4 serum levels.

- Serum Fatty Acid Binding Protein 4 was measured using "Human FABP 4 (Fatty Acid Binding Protein 4, Adipocyte) ELISA Kit” Catalog No: E-EL-H0285. 96T

\subsection{Statistical methods}

The collected data was revised, coded, tabulated and introduced to a PC using Statistical package for Social Science (IBM Corp. Released 2011. IBM SPSS Statistics for Windows, Version 20.0. Armonk, NY: 
IBM Corp.). Data were presented and suitable analysis was done according to the type of data obtained for each parameter.

\subsubsection{Descriptive statistics}

Mean Standard deviation $( \pm$ SD) for numerical data. Frequency and percentage of non-numerical data.

\subsubsection{Analytical statistics}

Student T Test was used to assess the statistical significance of the difference between two study group means.

Chi-Square test was used to examine the relationship between two qualitative variables

The ROC Curve (receiver operating characteristic) provides a useful way to evaluate the sensitivity and specificity for quantitative diagnostic measures that categorize cases into one of two groups. The optimum cut off point was defined as that which maximized the AUC value. The area under the ROC curve (AUC) results were considered excellent for AUC values between 0.9-1, good for AUC values between 0.8-0.9, fair for AUC values between 0.7-0.8, poor for AUC values between 0.6-0.7 and failed for AUC values between $0.5-0.6$
N.B: $p$ is significant if $<0.05$ at confidence interval $95 \%$.

\section{Results}

The mean age of AGA patients was 36.6 years. 30 male patients represented $60 \%$, while 20 female patients represented $40 \%$. In addition to 30 healthy control subjects of matched age and gender.BMI did not differ significantly between both groups ( $\mathrm{p}$ value $=0.562$ ). AGA patients were significantly reporting higher frequency of positive family history when compared to control group ( $\mathrm{P}$ value $=0.016)$. No significant differences were found in smoking history between all studied groups $(\mathrm{P}$ value $=0.306$ ). Mean serum level of FABP4 was significantly higher in AGA patients $(5.0 \pm 1.6 \mathrm{ng} / \mathrm{ml})$ than control $(2.7 \pm 0.8 \mathrm{ng} / \mathrm{ml})$ ( $\mathrm{p}$ value $<0.001$ ) Table (1).

ROC of serum level of FABP4 was conducted for discrimination between AGA patients and control groups. Serum level of FABP4 showed fair AUC (AUC=0.757). At cut off value of $4.3 \mathrm{ng} / \mathrm{mL}$, sensitivity was $54 \%$, specificity was $86.7 \%$, PPV was $87.1 \%$, NPV was $53.1 \%$, accuracy was $66.3 \%$ Table ( 2).

Table (1) Sociodemographic data, risk factors and serum level of FABP4 between all studied groups.

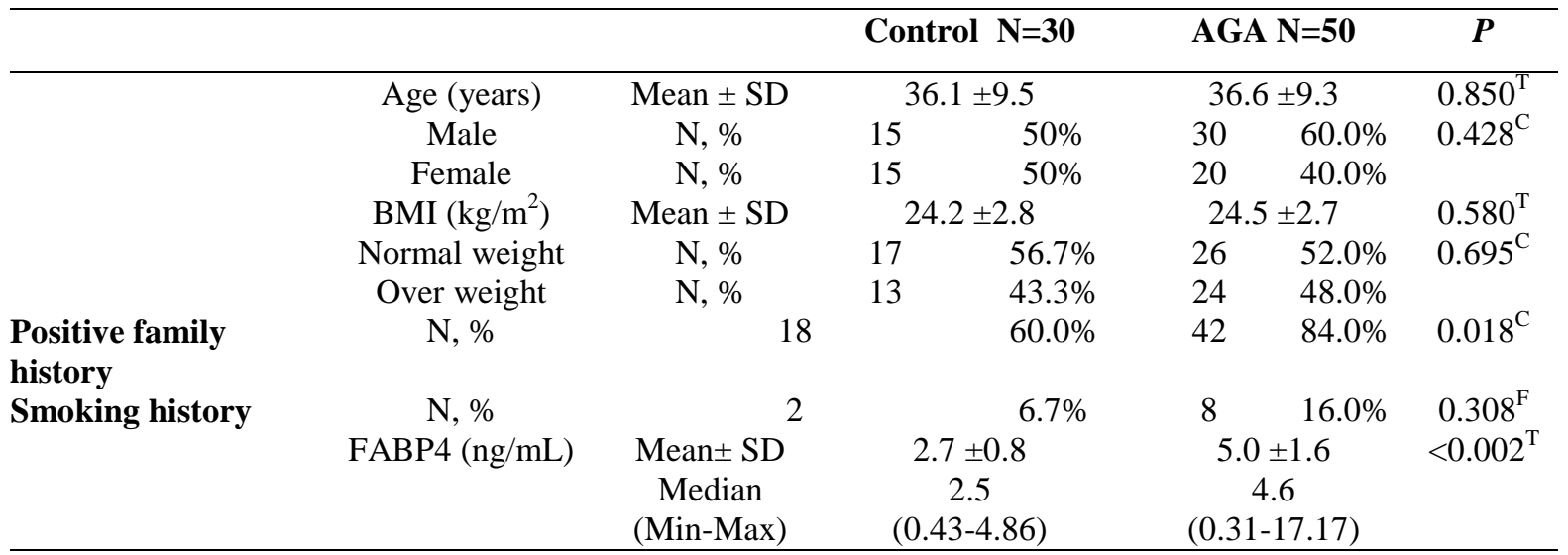

SD, standard deviation; T, Studentt test; C, chi square.

C, chi square; F, Fisher exact test.

$\mathrm{T}$, student $\mathrm{t}$ test

\section{Discussion}

To the best of our knowledge, there is not a single study in the published literature regarding the role of FABP4 in AGA.

Results of the present study revealed no significant difference between BMI values of AGA patients and the controls in the present study. Our study results are similar to Arias-Santiago et al. [4] and Mumcuoglu et al. [5] who could not find significant difference in BMI values of AGA cases and controls. However, Banger et al. [1] found out statistically higher BMI in AGA patients than controls, this may be due to most of their studied AGA patients were diabetics.
In current study and as reported in similar studies [6,1] family history of AGA was significantly high in study patients than controls.

The current results found that AGA patients showed significantly higher FABP4 levels when compared to controls.

Y. Zhang et al., [7] Suggested that due to the central roles of FABPs in facilitating fatty acid transport and metabolism, it is very likely that FABPs play a critical role in regulating obesity-induced inflammasome signaling.

S. Murphy et al., [8] suggested that disturbed lipid homeostasis could lead to organelle stress. The endoplasmic reticulum (ER), mitochondria, and 
peroxisomes are important sites for the metabolism of lipids [9] and [10] suggested that organelle stress induced by chronic disturbance of metabolic homeostasis in hair follicle cells could lead to the generation of reactive oxygen species by the ER and mitochondria, leading to activation of stress and inflammatory signaling cascades and oxidative damage. If ER and mitochondrial homeostasis were not restored, the organelles could activate apoptotic pathways in the hair follicle, destroying it.

Some histological studies illustrated perifollicular inflammation in the upper third of the hair follicles, suggesting that inflammation plays a pathogenic role in AGA, although clinically, AGA is considered a noninflammatory disease. Oxidative stress and inflammation are closely linked in biological systems so; there is also evidence of oxidative stress presence in dermal papilla cells of patients with androgenetic alopecia [11].

\section{Conclusion}

Serum FABP4 might play a role in AGA pathogenesis. Moreover, it could be considered a biomarker for early diagnosis of the disease and associated metabolic disorders with high sensitivity, specificity and accuracy rate.

\section{References}

[1] H.S. Banger, S.K. Malhotra, S. Singh, Is early onset androgenic alopecia a H.S. Banger, S.K. Malhotra, S Singh, Is early onset androgenic alopecia a marker of metabolic syndrome and carotid artery atherosclerosis in young Indian male patients? Int J Trichology, Vol.7(4), pp.141147,2018

[2] K.C. Dharam Kumar, Kishan Kumar YH and Neladimmanahally V () : Association of Androgenetic Alopecia with Metabolic Syndrome: A Case-control Study on 100 Patients in a Tertiary
Care Hospital in South India. Indian J Endocrinol Metab, vol. 22(2)pp.196-199,2018.

[3] S. Kralisch , M. Fasshauer ,Adiocyte fatty acid binding protein: a novel adipokine involved in pathogenesis of metabolic and vascular disease? Diabetologia, Vol.56(1)pp.10-21,2013.

[4] S. Arias-Santiago, M.T. Gutiérrez-Salmerón, Castellote-Caballero, Androgenetic alopecia and cardiovascular risk factors in men and women: A comparative study. J Am Acad Dermatol , Vol.63(3), pp.420-429,2010.

[5] C.Mumcuoglu, T.R. Ekmekci, S. Ucak The investigation of insulin resistance and metabolic syndrome in male patients with early-onset androgenetic alopecia. Eur J Dermatol, Vol.21(1), pp.79-82,2011.

[6] G. Assmann, H. Schulte , U. Seedorf , Cardiovascular risk assessment in the metabolic syndrome: Results from the prospective cardiovascular munster (PROCAM) study. Int J. Obes (Lond, vol.2, pp.S11-6,2008.

[7] Y. Zhang, Q. Li, E. Rao, Epidermal Fatty Acid binding protein promotes skin inflammation induced by high-fat diet. Immunity, vol. 42(5), pp.953-964,2015.

[8] S. Murphy, S. Martin , R.G. Parton, Lipid dropletorganelle interactions; sharing the fats. Biochim Biophys Acta Mol Cell Biol Lipids,vol.1791, pp.441-447,2009.

[9] D. Ron, P. Walter, Signal integration in the endoplasmic reticulum unfolded protein response. Nat Rev Mol Cell Biol, Vol.8, pp.519-529,2008.

[10]D.J. Todd, A.H. Lee , L.H. Glimcher, The endoplasmic reticulum stress response in immunity and autoimmunity. Nat Rev Immunol , Vol.8, pp.663-674,2008.

[11]B.E. Prie, L. Iosif, I. Tivig, Oxidative stress in androgenetic alopecia. J Med Life, Vol.9(1). Pp.79-83,2016. 